\title{
Control of baker's yeast fermentation: PID and fuzzy algorithms
}

\author{
Carlos Machado, Pedro Gomes, Rui Soares, Sílvia Pereira, Filomena O. Soares ${ }^{1}$
}

Dept. of Industrial Electronics, University of Minho, Portugal

Filomena.Soares@dei.uminho.pt

\begin{abstract}
A MATLAB/SIMULINK-based simulator was employed for studies concerning the control of baker's yeast fed-batch fermentation. Four control algorithms were implemented and compared: the classical PID control, two discrete versions- modified velocity and position algorithms, and a fuzzy law. The simulation package was seen to be an efficient tool for the simulation and tests of control strategies of the nonlinear process.
\end{abstract}

Keywords: baker's yeast fermentation, PID control, Fuzzy control.

\section{INTRODUCTION}

The building and use of mathematical models based on observed data is for long accepted as a basic scientific methodology. Models may be of a more or less formal character, but they have the basic feature that they attempt to link observations together into some pattern [1]. With the progress in digital technology, and thinking of bringing the theory into practice, computational modelling and modelbased applications have emerged and are recognized as areas of great priority [2].

The conventional approach for process modelling is based on the balance equations for mass, energy, and, if necessary, momentum and population. This form of modelling requires further knowledge about reaction kinetics, thermodynamic, transport and physical properties.

Real processes in the chemical, biochemical and food industry are in their vast majority non-linear MIMO systems. Their dynamics and control are difficult to study both for theoretical and practical reasons. In many instances experiments with real industrial processes are not carried out for reasons of economy and safety. Often on-line measurements are not available or simply they are too expensive. The simulation procedure represents an important tool to understand clearly the baker's yeast fermentation process.

\section{BAKER'S YEAST FERMENTATION - MODELLING APPROACH}

\section{A. Kinetic model}

Yeast growth is characterized by three metabolic pathways, viz-

$$
\begin{aligned}
& \mathrm{S}+\mathrm{C} \stackrel{\mu_{s}^{o}}{\rightarrow} \mathrm{X}+\mathrm{G} \\
& \text { (respiratory growth on glucose) } \\
& S \stackrel{\mu_{s}^{r}}{\rightarrow} \mathrm{X}+\mathrm{E}+\mathrm{G} \\
& \text { (fermentative growth on glucose) } \\
& E+\mathrm{C} \stackrel{\mu_{e}^{o}}{\rightarrow} \mathrm{X}+\mathrm{G} \\
& \text { (respiratory growth on ethanol) }
\end{aligned}
$$

where $\mathrm{S}$ represents glucose; O oxygen; X biomass; $\mathrm{E}$ ethanol; $\mathrm{C}$ carbon dioxide and $\mu_{s}^{o}, \mu_{s}^{r}, \mu_{e}^{o}$ : specific growth rates for the three pathways. In the sequel $\mathrm{X}, \mathrm{S}, \mathrm{E}$, $\mathrm{O}, \mathrm{C}$ mean concentrations.

The metabolic pathways of fermentative growth on glucose and oxidative growth on ethanol are competitive. This competition is governed by the respiratory capacity of the cells. If the instantaneous oxygen uptake capacity exceeds the oxygen need for total respiratory glucose uptake, then, all sugar uptake follows the respiratory pathway (1) with the remaining oxygen being spent on ethanol respiratory uptake (3). Otherwise, if the instantaneous oxygen uptake capacity is not enough, then, part of glucose uptake follows the respiratory pathway (1) while the remaining follows the fermentative pathway (2).

The kinetic scheme employed is based on the model proposed by [3]. Details on the reaction scheme, as well as the relevant kinetics data, including the yield coefficients, are presented and summarized elsewhere [4].

\footnotetext{
${ }^{1}$ Corresponding author.
} 


\section{B. Mechanistic model}

The mechanistic model for the fed-batch fermentation is obtained from mass balances for all components, considering that the reactor is well mixed. Furthermore it is assumed that the yield coefficients (Y) are constant and the dynamics of the gas phase can be neglected. Then the set of model equations is -

$$
\begin{aligned}
& \frac{d X}{d t}=\left(\mu_{s}^{o}+\mu_{s}^{r}+\mu_{e}^{o}-D\right) X \\
& \frac{d S}{d t}=\left(-\frac{\mu_{s}^{o}}{Y_{X / S}^{o}}-\frac{\mu_{s}^{r}}{Y_{X / S}^{r}}\right) X+\left(S_{f}-S\right) D \\
& \frac{d E}{d t}=\left(\frac{\mu_{s}^{r}}{Y_{X / E}^{r}}-\frac{\mu_{e}^{o}}{Y_{X / E}^{o e}}\right) X-D E \\
& \frac{d O}{d t}=\left(-\frac{\mu_{s}^{o}}{Y_{X / O}^{o}}-\frac{\mu_{e}^{o}}{Y_{X / O}^{o e}}\right) X-D O+O T R \\
& \frac{d C}{d t}=\left(\frac{\mu_{s}^{o}}{Y_{X / C}^{o}}+\frac{\mu_{s}^{r}}{Y_{X / C}^{r}}+\frac{\mu_{e}^{o}}{Y_{X / C}^{o e}}\right) X-D C-C T R
\end{aligned}
$$

where $\mathrm{D}$ is the dilution rate $(=F / V$, ratio feed rate/volume) and $S_{f}$ is the substrate concentration in the feed.

The accumulation of the working volume during the fedbatch process is represented by -

$$
\frac{d V}{d t}=D V
$$

The gas transfer rates are given by -

$$
\begin{aligned}
& O T R=K_{L}^{O} a\left(O^{*}-O\right) \\
& C T R=K_{L}^{C} a\left(C-C^{*}\right)
\end{aligned}
$$

where $K_{L}^{i} a$ are overall mass transfer coefficients for oxygen and carbon dioxide and $\mathrm{O}^{*}$ and $\mathrm{C}^{*}$ are the corresponding equilibrium concentrations.

\section{CONTROL ALGORITHMS}

In baker's yeast fed-batch fermentation ethanol production should be avoided. The ethanol accumulation may reduce the yield and the biomass productivity.

Nevertheless, it is desirable a small ethanol production in order to induce the enzymatic system of the fermentative pathway and then avoid a long period of adaptation when using the yeast [5].
The control algorithms presented for baker's yeast production referred to mono-variable control of dissolved ethanol concentration. The control action, in this case, is the flow of substrate feeding, F. The controller will have to maintain the ethanol set point in the constant value of 0.5 $\mathrm{g} / 1$.

Four control algorithms were implemented and compared: the classical PID control, two version of the corresponding discrete version: modified velocity and position algorithms, and a fuzzy law.

Figure 1 shows the PID controllers programmed in SIMULINK.

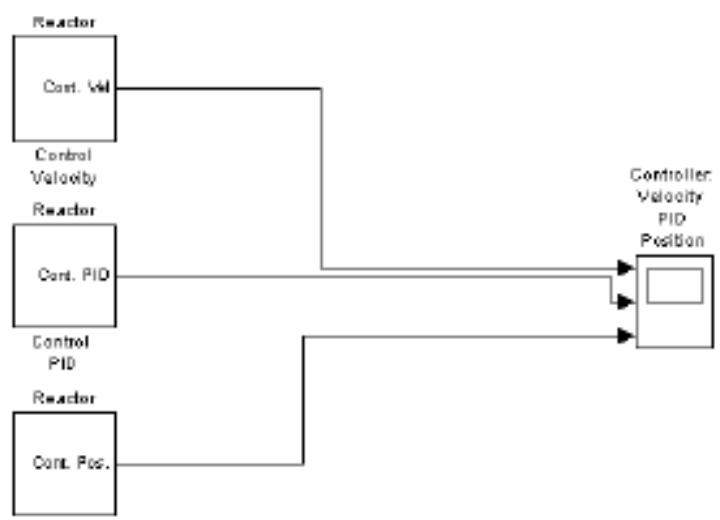

Figure 1 - SIMULINK program for PID controllers

Fuzzy control has been implemented in biotechnological processes, in particular in baker's yeast production. This methodology does not require accurate mathematical models for the process in study. But, for control it needs an accurate knowledge of the process in order to properly establish the rules [4].

\section{A. Continuous PID}

The mathematical equation that defines PID controllers is:

$F(t)=F_{e s t}+K_{c}\left(\operatorname{Err}(t)+\frac{1}{\tau_{i}} \int \operatorname{Err}\left(t^{\prime}\right) d t^{\prime}+\tau_{d} \frac{d E r r}{d t}\right)$

where:

$K_{C} \quad$ proportional gain of the controller

$\tau_{i} \quad$ integral time constant

$\tau_{d} \quad$ derivative time constant

Fest control action in steady state

Err error, difference between the set point and the output variable, $E r r=E_{r e f}-E$ (E means ethanol)

$F \quad$ control action (flow, in this case) 
In the three cases of classical PID (continuous or digital versions), controller PI was chosen, in opposition to the classic controller's complete version The reason is that, in fact experiments made in a laboratory where there are several electric equipments, it necessarily exists noise, and it is very well-known the great sensibility of the derivative action to the noise of the sign, particularly in discrete algorithms. The selected parameters were then: $\mathrm{K}_{\mathrm{c}}=\mathrm{lh}^{1} / \mathrm{gl}^{-1}$ and $\tau_{\mathrm{i}}=0.5 \mathrm{~h}$.

\section{B. Digital PID - velocity algorithm}

In the present work was implemented a version of the digital PI controller in the form of modified velocity [6]. In this situation the controller's equation is rewritten as:

$$
F_{n}=F_{n-1}+K_{c}\left(\left(E_{n-1}-E_{n}\right)+\frac{T}{\tau_{i}} E_{r r n}\right)
$$

One problem not yet referred of digital controller is the derivative kick [7]. When a variation in set-point is applied the control algorithm produces an abrupt change in the output signal. One method to avoid this unnecessary change is to substitute the error by $\mathrm{E}_{\mathrm{rr}}=\mathrm{E}_{\mathrm{ref}}-\mathrm{E}$.

The algorithm of modified velocity was implemented (Figure 2), representing its equation through a diagram of blocks in SIMULINK language. It was used, also, a "saturation" block to limit the inferior value of the flow to zero.

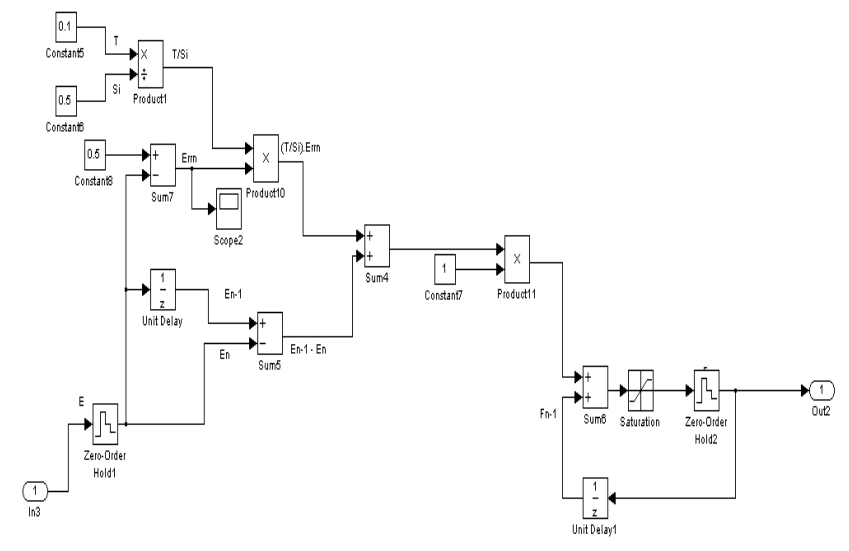

Figure 2 SIMULINK program for digital PID controller: modified velocity algorithm

\section{Digital PID - position algorithm}

It was also implemented a version of the digital PI controller in the form of position. In this situation the controller's equation is rewritten as:

$$
F_{n}=F_{e s t}+K_{c}\left(E r r_{n}+\frac{T}{\tau_{i}} \sum_{k=1}^{n} E r r_{k}\right)
$$

\section{Fuzzy controller}

The controller output is the valve opening that controls the glucose flow that enters in the fermertor. This controller has as inputs the ethanol level and the amount of existent biomass in the fermentor. The time of sampling is of 6 minutes, producing a different output in the same period. When there is a high glucose concentration in relation to the amount of biomass, more ethanol is produced, taking the controller to reduce or even to cut the glucose flow. Along the time, the controller will have to compensate the glucose flow, increasing it gradually so that it can compensate the existence of more biomass.

Due to these and other interactions, this process is highly non linear.

a) Connection scheme

The Fuzzy Controller has as inputs Ethanol (E) and the amount of Biomass (X) and as output the glucose Flow (F). It is necessary to place a saturation block in order to avoid that a command of negative flow is given.

The controller is also between two retainers that simulate the sampling of the inputs and the zero order hold of the flow command (Figure 3).

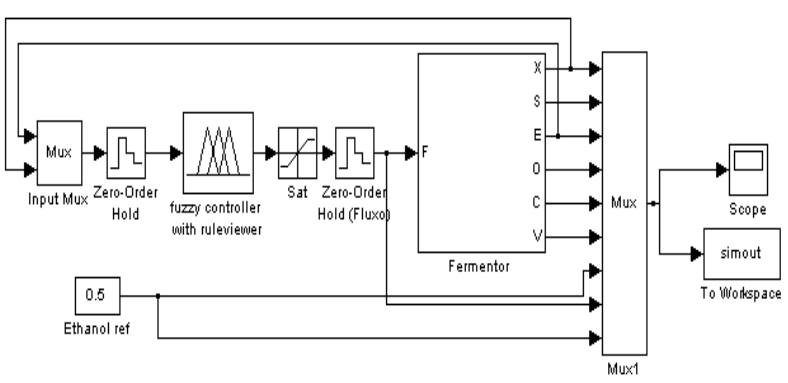

Figure 3 SIMULINK program for fuzzy controller

b) Type of the controller and parameters

The method chosen for the Fuzzy controller was SUGENO. In this method, the output variable is a group of finite values in a function $\mathrm{f}(\mathrm{u})$. This method is easier to tune in and also tends a low weight computational [8].

For the aggregation of the inputs in the operation AND it was chosen the method PROD, relating the several inputs simultaneously. For the OR aggregation it was chosen the method MAX, selecting the most important inputs.

In the defuzzification the sum was used for all the values of $f(u)$ to determine the value of the final flow (Figure 4). 


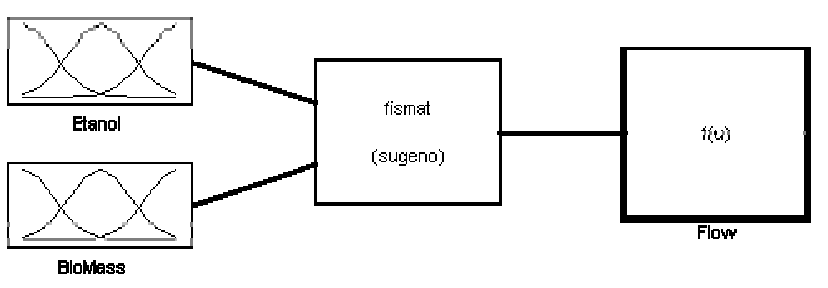

Figure 4 Defuzzification method used to obtain the final flow

\section{c) Fuzzification}

In the fuzzification procedure it is necessary to have in bill the maximum and minimum values. These maximum and minimum values define the zone where the controller is going to actuate. Out of those intervals, it is user's responsibility to guarantee that the output tends inside of the interval's values. There are two input variables: ethanol and biomass. Ethanol is the variable that we intend to control. Thus, we can see in Figure 5 three functions for this input: Low, Normal and High. Each of these has the maximum value where the state is true. Thus, for example in the Normal function, the truest state is in 0.5 that represents in our case the value to maintain (Reference).

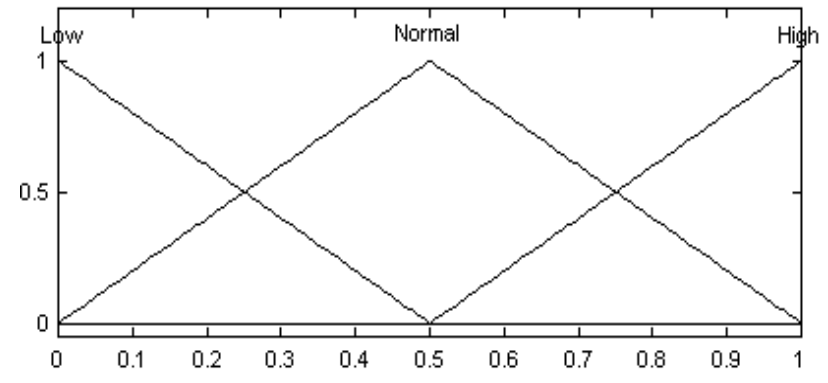

Figure 5 Ethanol fuzzification

Biomass is an input that is just good to compensate the output. Thus, with this entrance the flow is increasing in relation to Biomass (Figure 6).

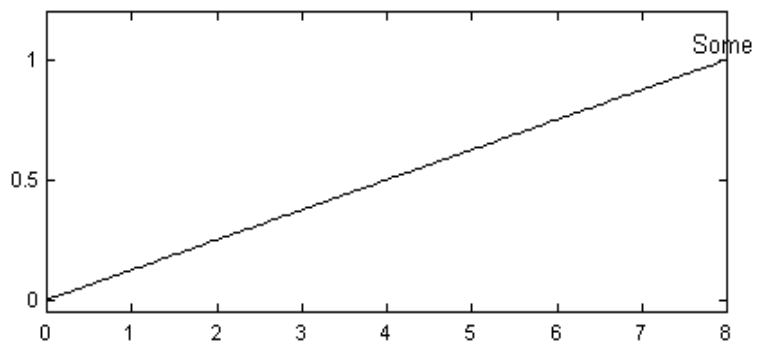

Figure 6 Biomass fuzzification

d) Defuzzification

In the defuzzification there are four constants that define the output because the controller is of the type SUGENO.

These constants are balanced, having as function the calculation of the controller's output. This controller still needs a saturation block to avoid negative flows given by the "Smaller" constant. The "Elevated", "Normal" and "Minor" constants defines the controller's normal operation. The "Larger" constant is the equivalent to an offset that will be useful when the error is low and the controller needs one final adjustment.

\section{e) Control Rules}

The control rules define the relationship between the inputs and the outputs. A rule calculates the weight of a certain output. The final output is the average of all the outputs.

The rules employed are:

- IF (ETHANOL IS HIGH) THEN (FLOW IS LOWER)

- IF (ETHANOL IS NORMAL) AND (BIOMASS IS SOME) THEN (FLOW IS HIGHER)

- IF (ETHANOL IS LOW) AND (BIOMASS IS SOME) THEN (FLOW IS HIGH)

The first rule is to lower the ethanol level, lowering the flow of entrance of glucose. The second rule happens when the ethanol is closed to the reference (normal) and the biomass concentration starts to grow, increases the inlet flow lightly. The last rule has the larger weight when the ethanol level is below the reference, still increases its effect in the existence of some biomass, placing a high flow.

\section{f) Tuning}

The tuning of one fuzzy controller can be quite complex, and there are several methods falling back upon the neural networks. A fuzzy controller can be tuned in through the parameters of fuzzification/defuzzification and/or by the rules of control. Due to the fact that it exists many calibration possibilities, this turns the tuning in a quite complex process. To turn the tuning easier, there are defined little rules (empirical rules) and some fuzzification parameters, just taking in consideration the limits of variation of the inputs. In the output there are three values (Smaller, Normal and Higher) defined with a logic that guarantees the convergence of the value of ETHANOL. Finally, a fourth value is included (output Higher), for controller's tuning.

With this method it becomes easier to tune in, therefore it is changed only one variable, being maintained the logic of convergence.

\section{SIMULATION PROCEDURE}

As the objective of this work is to control the addition of substrate in order to maintain a high productivity, being avoided the revenue loss by glucose excess in the middle and consequent ethanol production, a simulation program of 
baker's yeast production was developed in MATLAB/SIMULINK workspace.

With the objective of knowing the behaviour of the baker's yeast fermentation in relation to the external disturbs which can be decisive on the growth of biomass, we made a study of the influence, in open cycle of one variation of the feed flow substrate. Simultaneously this study allows the analyses of the influence of this change on the state variables.

Later a closed loop was implemented in order to test and compared the four control algorithms referred.

For comparing performances of the different control algorithms all the runs were taken at the same conditions, namely-

Control Objective: ethanol regulation for a set point of $E_{\mathrm{ref}}=0.5 \mathrm{~g} / \mathrm{l}$.

\section{Initial conditions:}

$$
\begin{aligned}
& \mathrm{X}(0)=0.3 \mathrm{~g} / \mathrm{l}, \mathrm{S}(0)=0.02 \mathrm{~g} / \mathrm{l}, \mathrm{E}(0)=0.5 \mathrm{~g} / \mathrm{l} \\
& \mathrm{O}(0)=.0066 \mathrm{~g} / \mathrm{l}, \mathrm{C}(0)=0.002 \mathrm{~g} / \mathrm{l}, \mathrm{V}(0)=2 \mathrm{~g} / 1, \\
& \mathrm{~S}_{\mathrm{f}}=30 \mathrm{~g} / 1
\end{aligned}
$$

Final volume: 51

Measured variable: Ethanol, E.

Controlled variable: Glucose feed-rate, F.

\section{RESULTS}

The Figures 7, 8, 9 show the obtained results with the three PID implemented algorithms: continuous PID, modified velocity algorithm and position, respectively.

We can conclude that there are no significant differences between the three PID versions.

Analysing the Figures 7 to 9 we can see that until $4 \mathrm{~h}$ 30m:

- the volume keeps constant, which means, equal to the initial volume (2 litters); this is due to the fact that the control if off because the initial concentration of ethanol is higher than the reference;

- the biomass growths from 0.3 (initial value) until approximately $0.85 \mathrm{~g} / \mathrm{l}$;

- the ethanol whose initial value was $0.8 \mathrm{~g} / \mathrm{l}$ reaches its maximum value $-0.96 \mathrm{~g} / \mathrm{l}$ about $1.3 \mathrm{~h}$, in the scale of time; value starting from which begins to decrease, once no longer glucose exists in the medium and the ethanol is consumed by the biomass.

After $4 \mathrm{~h} 30 \mathrm{~m}$ of simulation time it is verified that-

- at $16.6 \mathrm{~h}$ for position algorithm, $15.5 \mathrm{~h}$ for velocity and $15.7 \mathrm{~h}$ for continuous PID, the volume reaches its maximum (5 1) and for that, there is no more introduction of flow in the reactor;

- at approximately $16.2 \mathrm{~h}$ for position version and 16.4 $\mathrm{h}$ for PID, the concentration of ethanol reaches a null value and it maintains that value until the end of the simulation;

- at $16.7 \mathrm{~h}$ for position algorithm, $16.2 \mathrm{~h}$ for velocity and $16.3 \mathrm{~h}$ for continuous PID, the difference of the ethanol in relation to its reference value is 0 , that is to say the error is zero (ideal situation);
- in the end, the concentration of biomass in the reactor is approximately $9.29 \mathrm{~g} / \mathrm{l}$ for all controller versions.

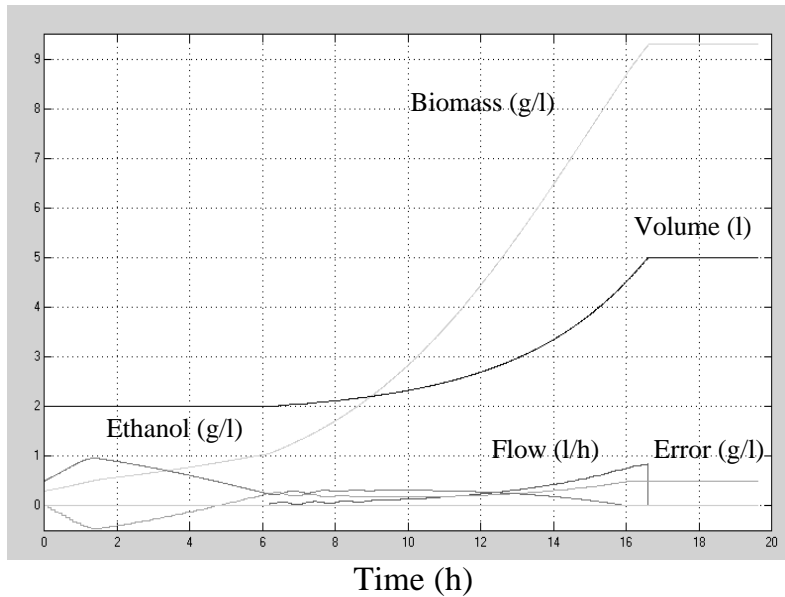

Figure 7 Continuous PID controller

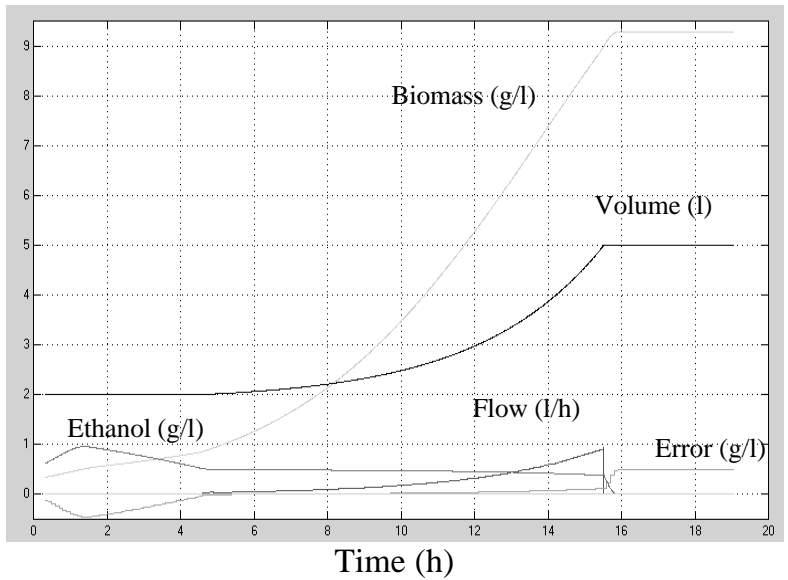

Figure 8 Modified velocity algorithm

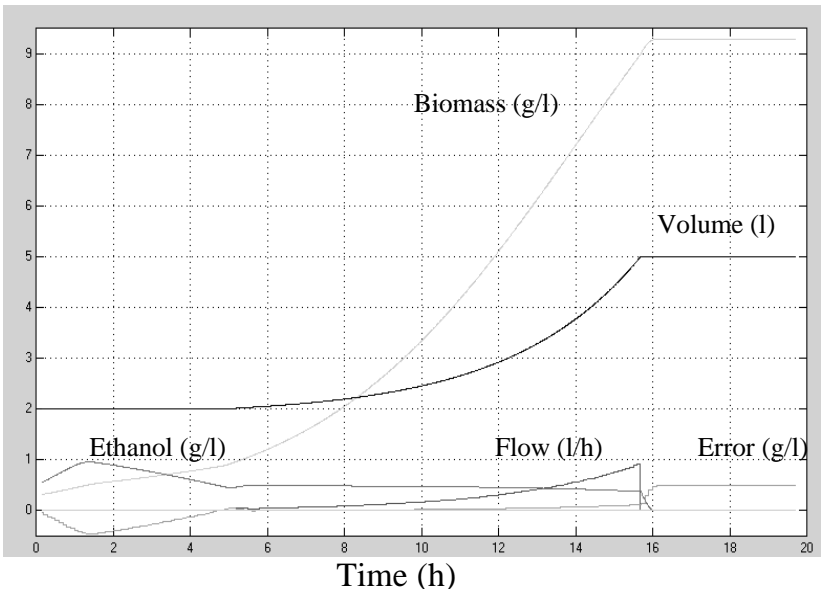

Figure 9 Position algorithm 
In Figure 10 there is the result obtained with the application of the Fuzzy controller. The ethanol concentration manages to follow set point but at the end it has still an offset. For the first six hours the control is off as the ethanol concentration is higher than the reference.

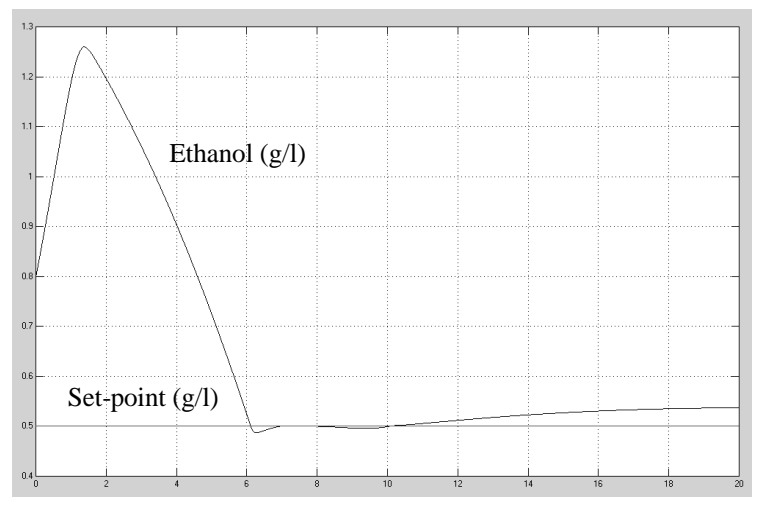

Time (h)

Figure 10 Fuzzy controller

\section{CONCLUSIONS}

A MATLAB/SIMULINK based simulator was developed for studies of fed-batch baker's yeast fermentation. Baker's yeast was chosen as a case study for its non-linearity.

The process was implemented and studied first in openloop mode at constant feed rate of glucose substrate $(\mathrm{F}=0.12$ $1 / \mathrm{h})$. Then a feedback control strategy was employed and PID and Fuzzy laws were compared. Concerning classical control three algorithms were programmed: the continuous version, the digitalized modified velocity form and the position algorithm.

Until $4: 30 \mathrm{~h}$, the application of different control algorithms does not show any difference because ethanol concentration is higher than the set-point value and, consequently the control is off.

Using the algorithm of modified position, we verified that the concentration of the ethanol has a much more unstable behaviour than using one of the other algorithms of control. The position algorithm constitutes an absolute control as we can see from the equation 14 .

We verified that when the maximum volume is reached, the biomass continues however to grow due to the ethanol that still exists in the reactor and is being consumed in that moment.

The fuzzy controller is especially useful when it is necessary to control an output with several input variables, because it is possible to define rules that relate the inputs and the corresponding output. Thus, the rules allow embedding in the controller the knowledge that is had of the process.

In spite of everything, the great disadvantage of the fuzzy controller is in the tuning, because it has many variables that can be altered and many connection methods in the rules. It's necessary to have some experience in fuzzy for taking real advantages of the controller.

It can be said that, in this case study, PID controller presents better acting. Of noticing is that, this conclusion has as base the results obtained in the simulations, where the effort applied in the controllers' tuning was similar. The Fuzzy Controller demands a deeper knowledge of the process in study and of the own operation of the controller.

We thought that the robustness and the tuning simplicity and PID controllers' implementation are the main reasons for the wide implementation of this type of controller in the industry.

\section{ACKNOWLEDGEMENTS}

The paper results from a work developed under the discipline Computer Control, included in the Industrial Electronics Master Course of University of Minho. The authors gratefully acknowledge the contributions of the colleagues Liliana Barbosa, Fernanda Moreira, Lurdes Gomes, Dalila Durães, Emílio Couto, Alberto Costa, Sara Torres, Manuel Romero.

\section{REFERENCES}

[1] Ljung, L., 1987, System Identification - Theory for the User, Prentice-Hall, New Jersey

[2] Edgar, T. F., 1996, Modelling and Control - Back to the Future, Part I, CAST Communications 19 (1), 7-12

[3] Sonnleitner, B., Käppeli, O., 1986, Growth of Saccharomyces cerevisiae is controlled by its Limited Respiratory Capacity: Formulation and Verification of a Hypothesis. Biotech. Bioneg., 28, 927-937

[4] Oliveira Soares, F., 1997, Monitorização $e$ controlo de fermentadores - Aplicação ao fermento de padeiro, Ph.D. Thesis, Universidade do Porto

[5] Pomerleau, Y. ,1990, Modélisation et Contrôle d'un Procédé Fed-Batch de Culture des Levures à Pain (Saccharomyces cerevisiae). PhD Thesis, Université de Montreal

[6] G. Franklin, J. Powell, M. Workman, Digital Control of Dynamic Systems, Addison-Wesley, ISBN 0201-33153-5, 1997.

[7] K. Astrom, B. Wittenmark, Computer Controlled Systems-Theory and Design, Prentice-Hall, ISBN 0-13172784-2, 1990.

[8] J.-S. R. Jang, C.-T. Sun E. Mizutani. Neuro-Fuzzy And Soft Computing - A computational approach to learning and machine intelligence. Prentice-Hall Inc, ISBN 0-13-261066-3, 1997 